\title{
High-redshift QSOs in GOODS
}

\author{
Fabio Fontanot*, Pierluigi Monaco
}

Astronomy Dep. Univ. of Trieste, via Tiepolo 11, I-34131 Trieste Italy

E-mail: fontanotets.astro.it

\section{Stefano Cristiani, Mario Nonino, Eros Vanzella}

INAF-Osservatorio Astronomico, via Tiepolo 11, I-34131 Trieste Italy

\section{and the GOODS AGN Team}

\begin{abstract}
Multiwavelenght surveys are a key instrument in detecting AGNs. AGNs are recognizable from their color properties and/or their infrared/X-ray emission. We discuss the recent developments of the GOODS survey and our selection of candidates based on optical color criteria and on the matching of the optical ACS database with infrared and X-rays counterparts. We pay particular attention to the high-z QSOs search in those fields. From these observation we obtain a sample of QSOs at redshifts from 3.5 to 5.2 and we put new constraints on the faint end of the Luminosity Function at those redshift, which is particularly important to understand the interplay between the formation of galaxies and super-massive black holes inside Dark Matter Halos and to measure the QSOs contribution to the UV ionizing background.
\end{abstract}

Baryons in Dark Matter Halos

5-9 October 2004

Novigrad, Croatia

${ }^{*}$ Speaker. 


\section{Introduction}

QSOs are intrinsically luminous and therefore can be seen rather easily at large distances; but they are rare, and finding them requires surveys over large areas. As a consequence, at present, the number density of QSOs at high redshift is not well known. Recently, the Sloan Digital Sky Survey (SDSS) has produced a breakthrough, discovering QSOs up to $z=6.43$ [7] and building a sample of six QSOs with $z>5.7$. The SDSS, however, has provided information only about very luminous QSOs $\left(M_{1450} \lesssim-26.5\right)$, leaving unconstrained the faint end of the high- $z$ QSO Luminosity Function (LF), which is particularly important to understand the interplay between the formation of galaxies and super-massive black holes (SMBH) and to measure the QSO contribution to the UV ionizing background [14]. New deep multi-wavelength surveys like the Great Observatories Origins Deep Survey (GOODS), described by [9], provide significant constraints on the space density of less luminous QSOs at high redshift. Here we present a search for high- $z$ QSOs, identified in the two GOODS fields on the basis of deep imaging in the optical (with HST) and X-ray (with Chandra), and discuss the allowed space density of QSOs in the early universe, updating the results presented in [5]. In the second part we present preliminary results on the search for high- $z$ QSOs, based on optical and infrared imaging.

\section{The Database}

The optical data used in this search have been obtained with the ACS onboard HST, as described by [9]. Mosaics have been created from the first three epochs of observations, out of a total of five, in the bands $F 435 W\left(B_{435}\right), F 606 W\left(V_{606}\right), F 775 W\left(i_{775}\right), F 850 L P\left(z_{850}\right)$. The catalogs used to select high- $z$ QSOs have been generated using the SExtractor software, performing the detection in the $z_{850}$ band and then using the isophotes defined during this process as apertures for photometry in the other bands. This is a common practice avoiding biases due to aperture mismatch coming from independent detections.

The X-ray observations of the HDF-N and CDF-S consist of 2 Ms and 1 Ms exposures, respectively, providing the deepest views of the Universe in the $0.5-8.0 \mathrm{keV}$ band. The X-ray completeness limits over $90 \%$ of the area of the GOODS fields are similar, with flux limits $(\mathrm{S} / \mathrm{N}=5)$ of $\approx 1.7 \times 10^{-16} \mathrm{erg} \mathrm{cm}^{-2} \mathrm{~s}^{-1}(0.5-2.0 \mathrm{keV})$ and $\approx 1.2 \times 10^{-15} \mathrm{erg} \mathrm{cm}^{-2} \mathrm{~s}^{-1}(2-8 \mathrm{keV})$ in the HDF-N field, and $\approx 2.2 \times 10^{-16} \mathrm{erg} \mathrm{cm}^{-2} \mathrm{~s}^{-1}(0.5-2.0 \mathrm{keV})$ and $\approx 1.5 \times 10^{-15} \mathrm{erg} \mathrm{cm}^{-2} \mathrm{~s}^{-1}$ $(2-8 \mathrm{keV})$ in the CDF-S field. The sensitivity at the aim point is about 2 and 4 times better for the CDF-S and HDF-N, respectively. As an example, assuming an X-ray spectral slope of $\Gamma=2.0$, a source detected with a flux of $1.0 \times 10^{-16} \mathrm{erg} \mathrm{cm}^{-2} \mathrm{~s}^{-1}$ would have both observed and rest-frame luminosities of $8 \times 10^{42} \mathrm{erg} \mathrm{s}^{-1}$, and $3 \times 10^{43} \mathrm{erg} \mathrm{s}^{-1}$ at $z=3$, and $z=5$, respectively (assuming no Galactic absorption). [1] produced point-source catalogs for the HDF-N and CDF-S and [8] for the CDF-S with more than 1000 detected sources in total.

\section{The Selection of the QSO candidates}

We have carried out the selection of the QSO candidates in the magnitude interval $22.45<$ $z_{850}<25.25$. Four optical criteria have been tailored on the basis of typical QSO SEDs in order to 
select QSOs at progressively higher redshift in the interval $3.5 \lesssim z \lesssim 5.2$.

$$
\begin{aligned}
& i-z<0.35 \text { AND } 1.1<B-V<3.0 \text { AND } V-i<1.0 \\
& i-z<0.35 \text { AND } B-V>3.0 \\
& i-z<0.5 \text { AND } B-V>2.0 \text { AND } V-i>0.8 \\
& i-z<1.0 \text { AND } V-i>1.9
\end{aligned}
$$

The criteria have been applied independently and produced in total 645 candidates in the CDF$\mathrm{S}$ and 557 in the HDF-N. They select a broad range of high- $z$ AGN, not limited to broad-lined (type-1) QSOs, and are less stringent than those typically used to identify high- $z$ galaxies. We have checked the criteria against QSOs and galaxies known in the literature within the magnitude and redshift ranges of interest confirming the high completeness of the adopted criteria: in figure 1 (left panel) we show a section of our color space with the limits corresponding to out color criteria. We superimpose the theoretical locus of QSOs, Ly-break and Seyfert2 galaxies at different redshifts, derived from the SEDs of [16] and [2], respectively.

Below $z \simeq 3.5$ the typical QSO colors in the ACS bands move close to the locus of stars and low-redshift galaxies. Beyond $z \simeq 5.2$ the $i-z$ color starts increasing and infrared bands would be needed to identify QSOs efficiently with an " $i$-dropout" technique.

\section{Match with Chandra Sources}

The optical candidates have been matched with X-ray sources detected by Chandra within an error radius corresponding to the $3 \sigma \mathrm{X}$-ray positional uncertainty. With this tolerance the expected number of false matches is five and indeed two misidentifications, i.e. cases in which a brighter optical source lies closer to the X-ray position, have been rejected (both in the CDF-S).

The sample has been reduced in this way to 11 objects in the CDF-S and 6 in the HDF-N. Type-1 QSOs with $M_{1450}<-21$, given the measured dispersion in their optical-to-X-ray flux ratio [18], are detectable in our X-ray observation up to $z \gtrsim 5.2$. Conversely, any $z>3.5$ source in the GOODS region detected in the X-rays must harbor an $\operatorname{AGN}\left(L_{x}(0.5-2 \mathrm{keV}) \gtrsim 10^{43} \mathrm{erg} \mathrm{s}^{-1}\right)$.

\section{Redshifts of the QSO candidates}

The GOODS team is developing a spectroscopic survey [17]. During this survey some of our candidates had a reduced spectrum, in addition to one spectroscopically confirmed QSO (HDF-N $123647.9+620941, z=5.186$ ), up to a total of twelve objects out of the 17 selected with a spectroscopic confirmation. Nine are QSOs with redshifts between 2.6 and 5.2. Three are reported to be galaxies, and the relatively large offsets between the X-ray and optical positions suggest that they could be misidentifications. In particular one object turn out to be a QSOs with a redshift greater than 4 (HDF-S 033229.29-275619.3, $z=4.76$ ). Both quasars of Type I and II are detected.

Photometric redshifts of the remaining QSO candidates have been estimated by comparing with a $\chi^{2}$ technique (see [2] for details) the observed ACS colors to those expected on the basis of $a$ ) the typical QSO SEDs; $b$ ) a library of template SEDs of galaxies (the "extended Coleman" of [2]). For the nine QSOs with spectroscopic confirmation the photometric redshifts are in good agreement with the observed ones. In general the estimates $a$ ) and $b$ ) are similar, since the color 



Figure 1: Left Panel: Expected $i_{775}-z_{850}$ vs. $B_{435}-V_{606}$ colors of QSOs as a function of redshift compared to other classes of objects. The dashed line shows the locus of QSOs at $z \gtrsim 3.5$ estimated with the composite spectrum of CV90. The continuous and dotted lines show the colors of Ly-break and Sy 2 galaxies, as computed from template SEDs. The corresponding symbols - open triangles, circles and squares - start on the left at $z=3.5$ and move to the right in steps of $\Delta z=0.1$. The observed colors of five QSOs are marked with filled circles and the corresponding redshifts. The same is done for ten high- $z$ galaxies identified with crosses. The positions of various types of stars is also shown. The dot-dashed line represents the projection of the selection criteria 1$)+3)$.Right panel: High-redshift $(z \gtrsim 4)$ QSOs cumulative Luminosity Function. The dots refer to observations made by different authors: [12] and [12] (red squares); [6] (green stars); [19] (magenta triangles). The blue circles refer to the present work and represent two confirmed QSOs and a highly probable QSO candidate for which the photometric-redshift was adopted. In the figure we also shown a comparison of observational points with PLE (dashed line) and PDE (solid line) model (see text for more details).

selection criteria both for galaxies and QSOs rely on a strong flux decrement in the blue part of the spectrum - due to the IGM and possibly an intrinsic Lyman limit absorption - superimposed on an otherwise blue continuum. If we limit ourselves to the redshift range $z \gtrsim 4$ - where the selection criteria and the photometric redshifts are expected to be most complete and reliable - we estimate that one more QSOs could be present in the GOODS, depending on whether galaxy or QSO SEDs are adopted for the photometric redshifts. This brings the total to no more than three QSOs with redshift larger than 4 .

\section{High-redshift QSOs Luminosity Function}

In figure 1 (right panel) we present our estimate of the cumulative Luminosity Function for QSOs with $z \gtrsim 4$. Our observational points (blue circles) are compared with the results of other surveys. The agreement is quite good. We try also to compare our estimate with two phenomenological models. 
The double power-law fit of the 2QZ QSO LF [4] has been extrapolated for $z>2.7$ (the peak of QSO activity) in a way to produce a power-law decrease of the number of bright QSOs by a factor 3.5 per unit redshift interval, consistent with the $3.0_{-0.9}^{+1.3}$ factor found by [6] for the bright part of the LF. The extrapolation is carried out either as a Pure Luminosity Evolution (PLE) or a Pure Density Evolution (PDE). The PLE model predicts about 17 QSOs with $z_{850}<25.25$ at redshift $z>4$ (27 at $z>3.5$ ) in the $320 \operatorname{arcmin}^{2}$ of the two GOODS fields, and is inconsistent with the observations at a more than $3 \sigma$ level. The PDE estimate is 2.9 QSOs at $z>4(6.7$ at $z>3.5)$.

\section{Work in Progress}

We are exploring other techniques to select $z \gtrsim 4$ AGNs without making any assumption on the optical-to-x-ray flux ratio. In particular we have added to the optical selection (2)-(4) criteria based on the IR colors and/or on the morphology of the candidates (i.e. the request of a point-like appearance). In this way we have selected a sample of 5 candidates in the nothern field and in 5 candidates in the southern field. The two already known $z \gtrsim 4$ QSOs are recovered with this new selection and spectroscopic follow-up on the remaining ones is underway.

\section{Conclusions}

At $z \gtrsim 4$ the space density of moderate luminosity $\left(M_{1450} \simeq-23\right)$ QSOs is significantly lower than the prediction of simple recipes matched to the SDSS data, such as a PLE evolution of the LF. A flattening of the observed high- $z$ LF is required below the typical luminosity regime $\left(M_{1450} \lesssim-\right.$ 26.5) probed by the SDSS.

An independent indication that this flattening must occur comes from the statistics of bright lensed QSOs observed in the SDSS [20] that would be much larger if the LF remains steep in the faint end.

A similar result has been obtained at $5 \lesssim z \lesssim 6.5$, by [3]. The QSO contribution to the UV background is insufficient to ionize the IGM at these redshifts.

This is an indication that at these early epochs the formation or the feeding of SMBH is strongly suppressed in relatively low-mass $\mathrm{DMH}$, as a consequence of feedback from star formation [10] and/or photoionization heating of the gas by the UV background [11], producing a kind of inverse hierarchical baryon collapse.

\section{References}

[1] Alexander, D.M., et al. 2003, AJ 126, 539

[2] Arnouts, S., Cristiani, S., Moscardini, L., et al., 1999, MNRAS 310, 540

[3] Barger, A.J., Cowie, L.L., Capak, P., et al., 2003, ApJ 584, L61

[4] Boyle, B.J., Shanks, T., Croom, S.M., et al., 2000, MNRAS 317, 1014

[5] Cristiani, S., et al. 2004, ApJ 600, L119

[6] Fan, X., Strauss, M.A., Schneider, D., et al., 2001, AJ 121,54

[7] Fan, X., Strauss, M.A., Schneider, D.P., Becker, R.H., et al., 2003, AJ 125, 1649 
[8] Giacconi, R., et al. 2002, ApJS 139, 369, G02

[9] Giavalisco, M., et al. 2004, ApJ 600, L93

[10] Granato, G.L., Silva, L., Monaco, P., et al., 2001, MNRAS 324, 757

[11] Haiman, Z., Madau, P., \& Loeb, A., 1999, ApJ 514, 535

[12] Kennefick, J.D., Djorgovski, S.G., \& de Carvalho, 1995, AJ 110, 2553

[13] Kennefick, J.D., Djorgovski, S.G., \& Meylan, G., 1996, AJ 11, 1816

[14] Madau, P., Haardt, F., Rees, M.J., 1999, ApJ 514, 648

[15] Monaco, P., Salucci, P., Danese, L., 2000, MNRAS 311, 279

[16] Schmitt, H.R., Kinney, A.L., Calzetti, D., Storchi-Bergmann, T., 1997, AJ, 114, 592

[17] Vanzella, E., et al.2004, submitted to A\&A (astro-ph/0406591),

[18] Vignali, C., et al.2003, AJ 125, 2876

[19] Wolf, C., Wisotzki, L., Borch, A., et al., 2003, A\&A 408, 499

[20] Wyithe, J.S.B., Loeb, A., 2002, ApJ 577, 57 